\title{
LA RESPONSABILIDAD SOCIAL GUBERNAMENTAL Y SU INFLUENCIA EN LA CULTURA DE CALIDAD DEL GOBIERNO MUNICIPAL DE VILLAFLORES, CHIAPAS
}

\author{
M. D. SÁNCHEZ-FERNÁNDEZ ${ }^{1}$, M. RÍOS-MANRÍQUEZ ${ }^{2}$ e A. G.D. CORREIO \\ Universidade da Coruña ${ }^{1}$, Universidad de Guanajuato ${ }^{2,3}$ \\ msanchezf@udc.es ${ }^{1}$
}

Artigo submetido em 14/01/2019 e aceito em 14/05/2019

DOI: $10.15628 /$ holos.2019.8189

\section{RESUMEN}

En años recientes han surgido dos conceptos importantes, el primero es el de Responsabilidad social, el cual comprende a los individuos, las empresas y el gobierno, mientras que el segundo es el termino de Cultura de calidad, el cual se ha venido utilizando principalmente en las empresas, no obstante, también puede ser utilizado en cualquier tipo de organización humana, como es el caso de este estudio, que se enfoca en el gobierno. Este trabajo tiene por objetivo analizar cuál es la Influencia de la Responsabilidad Social Gubernamental en la Cultura de calidad del gobierno de Villaflores, Chiapas. Para lo cual se analizaron cinco variables o áreas de estudio que fueron La cultura de calidad, la Responsabilidad Social Gubernamental (RSG), la calidad de vida, Imagen gubernamental y la
Responsabilidad con los ciudadanos por parte del gobierno. Se realizó una prueba piloto en la cual se contó con la participación de 60 personas, el análisis fue mediante estadísticos descriptivos, tablas de contingencia, correlaciones a través de chi-cuadrado de Pearson y Regresión lineal múltiple. Se encontró que en general en las cinco áreas analizadas hay una percepción negativa por parte de los ciudadanos hacia la gestión del gobierno, lo cual no depende de si el encuestado es originario o no del municipio, en las correlaciones y la regresión lineal, se encontró que hay una relación positiva y significativa entre la cultura de calidad y la RSG, finalmente el modelo explicativo destaca que la RSG tiene una influencia positiva con la cultura de calidad del gobierno municipal de Villaflores, Chiapas.

PALABRAS CLAVE: Cultura de calidad; Responsabilidad Social Gubernamental; Calidad de vida; Imagen Gubernamental; Responsabilidad con el ciudadano.

\section{The Quality Culture and its influence on the Social Responsibility of the Municipal Government of Villaflores, Chiapas}

\section{ABSTRACT}

In recent years, two important concepts have emerged: the first is that of Social Responsibility, which includes individuals, companies and government, while the second is the term Quality Culture, which has been used mainly in the Business; however, it can also be used in any type of human organization, as in the case of this study, which focuses on government. The objective of this work is to analyze the influence of the Governmental Social Responsibility on Quality culture of the municipal government of Villaflores, Chiapas. For what can be analyzed five variables or areas of study that were Quality culture, Government Social Responsibility (GSR), the quality of life, the image of the government and the responsibility with the citizens in the part of the government. A pilot test was carried out in which 60
\end{abstract}

people participated, the analysis was through statistical descriptions, contingency tables, correlations through Pearson's chi-square and multiple linear regression. . It was found that in general in the five areas analyzed there is a negative perception on the part of citizens towards the management of the government, which does not depend on whetherthe respondent is from the municipality or not. In the correlations and linear regression, it is found that there is a positive and significant relationship between the Quality Cultureand the GSR, finally the explanatory modelemphasizes that the GSR has a positive influence on the quality culture of the municipal government of Villaflores, Chiapas.

KEYWORDS: Quality Culture, Government Social Responsibility, Quality of life, Image of the government, Responsibility with the citizens. 


\section{INTRODUCCIÓN}

La cultura organizacional es uno de los factores principales que apoya a todas aquellas organizaciones que quieren hacerse competitivas. Por esto, un punto importante en torno al cual gira este trabajo es el estudio de la cultura organizacional, especialmente la cultura de calidad y la Responsabilidad Social en el Gobierno municipal de Villaflores, Chiapas.

Actualmente en México es la organización dentro del Gobierno la que requiere emprender un cambio cultural que permita a adopción de la Responsabilidad Social (RS) no solo para cumplir con las obligaciones para con su personal sino también con sus grupos de interés, buscando generar el desarrollo laboral y científico de la sociedad.

En los últimos años el término Responsabilidad Social(RS) ha tomado importancia, ya que despierta la atención de empresas, analistas, críticos, consultores, gobierno y sociedad civil (Aguilar, 2013). Este estudio está enfocado en la responsabilidad social pública o gubernamental. Que un gobierno adopte este enfoque en sus acciones implica que habrá una mejora de la calidad democrática en las entidades públicas, las instituciones gubernamentales y por consiguiente en su administración (Ruíz-Rico, 2014).

La Responsabilidad social puede definirse como el compromiso que tienen las personas, ya sean miembros de una sociedad, de una empresa o incluso del gobierno de contribuir por voluntad propia para tener una sociedad justa y proteger el medio ambiente (Moneva, 2005; ISO26000; 2010; Cajiga, 2018). La responsabilidad social es el compromiso, consiente y congruente (Cajiga, 2018), que tienen las personas, miembros de una sociedad o asociados a una empresa de contribuir libremente para tener una sociedad más justa y proteger el ambiente (Moneva, 2005; ISO26000, 2010).

Mientras que, la cultura de calidad se puede definir como "esa cultura dentro de la organización que es conducida por los empleados; la solución de los problemas; orientado al cliente, abierto y sin temor, donde las prácticas comerciales de la organización se basan en la búsqueda de mejoras continuas, la delegación de decisiones, la eliminación de barreras funcionales, la eliminación de fuentes de error, el trabajo en equipo y la toma de decisiones basadas en hechos" (González-Ortiz, Escalera-Chávez, \& Pérez-Veyna, 2010, p.40).

Es así que la Responsabilidad Social Gubernamental (RSG) o pública, se puede definir como la capacidad que tienen los organismos gubernamentales de reaccionar ante los efectos que tienen sus acciones en los grupos con los que interactúa (Vélez-Romero y Cano-Lara, 2016). Aunque la RSG se considera un concepto ajeno al derecho, lo cual sucita cierto grado de escepticismo científico y jurídico (Ruiz-Rico, 2014), sí adquiere relevancia como respuesta a la constatación de una imagen degradada, en la que se pone especial atención en tres aspectos: medioambiental, social y económico (Rivero, 2006).

Actualmente los gobiernos de México tienen problemas de credibilidad, confianza e imagen (Aguilar, 2013), debido a una mala gestión y a la corrupción que persiste en el gobierno, que se ha 
venido desarrollando desde hace ya algún tiempo(Gordoa, 2008; Morris, 1992), y sobre todo a la falta de cumplimiento de la ley (Lomnitz-Adler, 2000; y Morris, 1992).

El estado de chiapas se encuentra ubicado en la región sureste de México, colinda al norte con el estado de Tabasco, al oeste con Veracruz y Oaxaca, al sur con el Océano Pacífico y al este con la República de Guatemala. Al norte $17^{\circ} 59^{\prime}$, al sur $14^{\circ} 32^{\prime}$ de latitud norte; al este $90^{\circ} 22^{\prime}$, al oeste $94^{\circ} 14^{\prime}$ de longitud oeste(Chiapas Gobierno del Estado, 2019), en la figura 1 se presenta un mapa de Chiapas.Por otra parte Villaflores es un municipio perteneciente a este estado, el cual tiene una extensión territorial de $1232.10 \mathrm{Km} 2$, limita al norte con Suchiapa, Jiquipilas y Ocozocoautla, al este con Chiapa de Corzo y Villacorzo, al sur con Villacorzo y Tonalá, al oeste con Jiquipilas y Arriaga (SCIM, 2018). En el ámbito económico, el $49.41 \%$ de la Población Económicamente Activa (PEA) se encuentra en actividades agropecuarias, el $12.51 \%$ se dedica a actividades secundarias y en el sector terciario se encuentra el $36.49 \%$ de la PEA (INEGI, 2018).

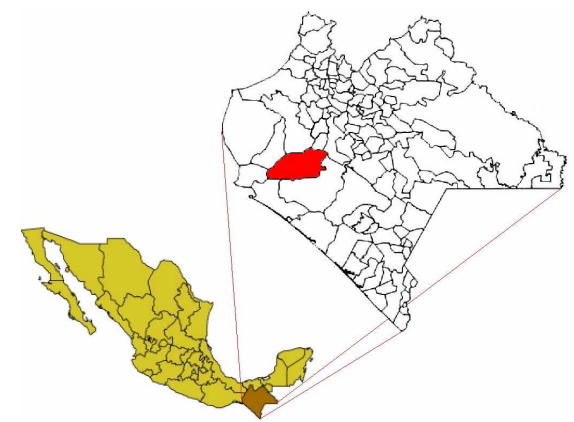

Figura 1. Mapa de ubicación del estado de Chiapas y el municipio de Villaflores

Fuente: Chiapas Gobierno del Estado (2019).

A nivel nacional, el $88 \%$ de los ciudadanos perciben la corrupción por parte del gobierno, (Instituto Nacional de Estadística y Geografía (INEGI, 2016); sin embargo, en el estado de Chiapas la percepción es de un $94 \%$, Solamente un $0.1 \%$ menos que el estado en el que más se percibe (INEGI, 2016). Dentro de las repercusiones que se llegan a tener a causa de la corrupción se encuentran la inseguridad y delincuencia; desempleo, pobreza y mal desempeño del gobierno (INEGI, 2016). Para el estado de Chiapas, en el ámbito económico se encontró que aporta el 1.7\% del PIB nacional (INEGI, 2016), también se encuentra ubicado en el lugar 31 de 32 en la tasa de participación económica del país (INEGI, 2018). En cuanto a los temas que le atañen a la RSG, se encontró que ocupa el 15vo lugar en contaminación del aire (INEGI, 2018), se ubica en los últimos tres lugares en disposición de residuos, con un $29 \%$ de actividad en este rubro, refiriéndose esto a que el $71 \%$ de los residuos no reciben un tratamiento adecuado para evitar la contaminación (INEGI, 2018), en cuanto a lo social, se encontró que el $85 \%$ de sus habitantes tienen acceso a servicios de salud, lo que lo coloca en el lugar 19 de los 32 estados federativos que conforman México (INEGI, 2018), y solo el $77.8 \%$ de los ciudadanos tienen viviendas con acceso a servicios públicos, lo cual indica que el $22.2 \%$ de los habitantes del estado no cuenta con los servicios públicos básicos, lo que lo posiciona en el lugar 28 de 32 (INEGI, 2018).

Navarro-Galera, et al. (2014) mencionan que hay muchas razones que muestran la oportunidad y el interés en la investigación de la sostenibilidad en el sector público; también se menciona que existen pocos estudios que se dediquen expresamente a la cuestión de la RS en las entidades públicas. Por otra parte, Wilson (2015), González-Ortizet al. (2010) y Gillies (2015) han demostrado que la cultura de calidad puede resolver y potenciar a una organización, en este caso 
el gobierno, ya que abarca problemas como la transparencia, el desarrollo de los grupos de interés, preocupación por mejorar la calidad de vida de los grupos interesados entre otras cosas. Sin embargo, dentro de la literatura revisada no se encontraron estudios que abarquen estos dos conceptos del gobierno.

El estudio de la RS pública o Gubernamental con la cultura de calidad podría influir positivamente en el nivel de compromiso por parte de los gobiernos municipales y la percepción que los ciudadanos tienen de estos para implementar políticas que sean socialmente responsables (Navarro-Galera et al, 2014). Los estudios sobre RS, especialmente en RSG pueden coadyuvar a que haya un desarrollo sostenible (ISO26000: 2010).

Motivos por los cuales resulta importante estudiar la Responsabilidad Social Gubernamental y la cultura de calidad, sobre todo, por los resultados obtenidos por el Instituto Nacional de Estadística y Geografía (INEGI, 2016), referentes a la mala percepción del ciudadano respecto a su Gobierno, por ello el objetivo es analizar cuál es la Influencia de la Responsabilidad Social Gubernamental en la Cultura de calidad del gobierno de Villaflores, Chiapas.

\section{MARCO TEÓRICO}

La RS es un producto social que busca el mutuo beneficio con todos los actores involucrados, lo que la liga con la cultura que persista en el objeto a estudiar (llámese empresa, individuo o Gobierno), de acuerdo con Diez (2007, P. 231)“La cultura y la responsabilidad social son dos fenómenos que componen la realidad sociocultural de las organizaciones, pues integran el enfoque estratégico que la organización estime a bien llevar a cabo".

\subsection{Cultura de Calidad}

Originalmente la cultura de calidad, al igual que otros paradigmas de la calidad, fue propuesta para alcanzar los objetivos internos que la Administración de la Calidad Total (TQM por sus siglas en inglés) requiere (Hairuddin y Mohammed, 2012), esta se introduce como un enfoque integral que hace sostenible la calidad de los productos o servicios(Ulf-Daniel, 2009), por ello se ha visto la posibilidad de expandir este termino y pueda ser usado en la organización que hay en el gobierno.

Una definición más completa es la desarrollada por González-Ortiz, et al., (2010) que dice que es "esa cultura dentro de la organización que es conducida por los empleados; la solución de los problemas; Orientado al cliente, abierto y sin temor, donde las prácticas comerciales de la organización se basan en la búsqueda de mejoras continuas, la delegación de decisiones, la eliminación de barreras funcionales, la eliminación de fuentes de error, el trabajo en equipo y la toma de decisiones basadas en hechos" (p. 40).

Se puede decir también que la cultura es el modelo por el cual los individuos pertenecientes a un grupo de personas o a una sociedad son educados e incorporados a la actividad de este (García-Álvarez, 2006; Anzola, 2005; Lo, 2002; Barney, 1986). 
Dentro del Gobierno la cultura de calidad crea, y a su vez es creada, por la calidad del medio ambiente interno; en consecuencia, condiciona el grado de cooperación y de dedicación de la institucionalización de propósitos dentro de una organización. Uno de estos propósitos es la Responsabilidad Social.

\subsection{Responsabilidad social}

Con la finalidad de entender este tema es importante definir la Responsabilidad Social (RS), para lo cual se usarán diferentes fuentes que han definido la RS, CENTRARSE (2018) define a la RS como una cultura de negocios basada en principios éticos y el firme cumplimiento de la ley, respetuosa de las personas, las familias, las comunidades y el medioambiente, que contribuye a la competitividad de las empresas, al bienestar general y al desarrollo sostenible del país.

La responsabilidad social es la responsabilidad que tiene una organización (ISO 26000, 2010) que se da de manera voluntaria (Aguilar, 2013), de reaccionar a los impactos que sus decisiones y actividades ocasionan a la sociedad y medioambiente (Cancino y Morales, 2008) a través de comportarse éticamente (Navarro-Galera et al., 2014) y ser transparentes (Rodríguez, 2008).

Otra forma de definir la RS es cómo una forma de compromiso (Cajiga, 2018) que se da de manera voluntaria con el desarrollo de la sociedad (Aguilar, 2013) y la preservación del medio ambiente (CENTRARSE, 2018), desde la estructura social a un comportamiento responsable hacia las personas (Moneva, 2005).

Para implementar la RS se ha tomado como base el comportamiento ético, ya que la ética sirve para regular parámetros de operación que buscan que las decisiones tomadas sean benéficas para las personas (Rodríguez, 2008). Respecto a esto la ISO26000 (2010) también explica que las organizaciones deben contribuir al desarrollo sostenible, debe tomar en cuenta las expectativas de las partes interesadas, y deben integrar la RS en toda la organización y sus relaciones internas.

La RS se puede medir a través de tres dimensiones: económica, social y ambiental (Cancino y Morales, 2008). En el ambito económico se pueden medir, cuando hablamos de la percepción de los encuestados, mediante la calidad de vida, los niveles de ingresos, entre otros.

Se entiende la Responsabilidad Social Gubernamental (RSG) como "la capacidad de respuesta que tienen las organizaciones gubernamentales frente a los efectos de sus acciones sobre los diferentes grupos con los que se relaciona, generalmente se puede decir que tiene una esencia socialmente responsable" (Vélez-Romero y Cano-Lara, 2016, p. 123).

La RSG está enfocada principalmente al diseño y ejecución de políticas públicas dirigidas a los grupos más vulnerables de la sociedad (Ruiz-Rico, 2014), lo cual conlleva a emitir leyes, decretos, programas sociales, etc., que repercutan sobre el entorno social en el que se rigen (VélezRomero y Cano-Lara, 2016; Navarro-Galera, et al., 2014).

Un gobierno que sea socialmente responsable debe asegurar la calidad de vida que tenga la sociedad en la que se rija (Vélez-Romero y Cano-Lara, 2016), esto quiere decir que la sociedad debe por lo menos tener buenos servicios (tanto públicos como privados), también debe ser transparente en su manejo de presupuesto y esta información debe estar siempre disponible para 
la sociedad (Cardona y Agudelo, 2005), por último, la RSG se limita en su campo de acción al marco legal que la rige, es decir a la legitimidad, con que hace las cosas (Hernández, 2009; Subirats, 1994).

\subsection{Imagen Gubernamental}

La imagen gubernamental se refiere a la imagen pública que el gobierno tiene. Una buena imagen por parte de sus gobernantes genera confianza en cada uno de los ciudadanos, Maquiavelo en su obra "el príncipe" fue uno de los primeros en poner las bases de la imagen publica y su importancia, y en trabajos posteriores como los de Abreu (1998), Gordoa (1999) y Aceves (2002) entre otros más han tomado como base su trabajo para dar importancia a la imagen publica que se maneja en el gobierno. Estos autores dicen que la importancia recae en que cuando se tiene una imagen publica positiva los gobiernos tienen mayor credibilidad y confianza de los habitantes. No obstante, hace falta saber que es la imagen pública.

La imagen pública se encuentra asociada con la percepción, o también llamada sensación interior la cual se trata de una impresión que se hace en los sentidos de las personas (Gordoa, 1999), cuando la imagen de una sola persona se comparte con otras personas se dice que ha evolucionado a una imagen mental colectiva (Abreu, 1998), es decir, la imagen pública es la percepción que se comparte en un grupo de personas la cual provoca una respuesta unificada (Aceves, 2002; Gordoa, 2008). Además, la imagen de un gobierno se debe cuidar y proteger ya que es un elemento fundamental para tener credibilidad ante las personas (López-Pinto, 2001).

Uno de los factores que influencia hacia tener una percepción positiva del gobierno es el que estos aseguren la calidad de vida de sus ciudadanos, es decir, que los habitantes de un lugar sientan que tienen las mejores condiciones para vivir.

\subsection{Calidad de vida de los ciudadanos}

El término calidad de vida ha ido evolucionando desde finales de la segunda guerra mundial, aunque aún era un término algo vago, para los años 80 se sabía que tenía que ver con la satisfacción de necesidades, en los noventa, la expresión se identificó en tres contextos y significados que son: descriptivo, evaluativo y prescriptivo (Cardona y Agudelo, 2005).

La organización mundial de la salud (OMS) define la calidad de vida como "la percepción que un individuo tiene del lugar en el que existe, en el contexto de la cultura y del sistema de valores en los que vive y en relación con sus expectativas, normas e inquietudes. Se trata de un concepto muy amplio que está influido de modo complejo por la salud física del sujeto, su estado psicológico, su nivel de independencia, sus relaciones sociales, así como su relación con los elementos esenciales de su entorno" (OMS, 1997, p.3).

"La calidad de vida es un concepto multidimensional y multidisciplinario que debe ser valorado desde la presencia de las condiciones materiales y espirituales que permitan el desarrollo psicobiológico y social-histórico del ser humano y la satisfacción personal con las condiciones de vida que cada persona ha logrado alcanzar" (Baldi y García, 2005, p. 9). Rueda (2004) menciona que el concepto de calidad de vida es impreciso, multidimensional y multidisciplinario, es decir que no existe una única teoría que defina y explique el fenómeno (Verdugo, 2013; Rueda, 2004). 
Una vez se tiene un poco más claro el concepto de calidad de vida, es necesario saber cómo se mide o cuales son los parámetros que se utilizan para medirla (Rodríguez, 2008; Rueda, 2004). Los indicadores de calidad de vida incluyen no solo elementos de riqueza y empleo sino también de ambiente físico, salud física y mental, educación, recreación y, lo que es muy importante, la sensación de pertenencia a una comunidad o la cohesión social (Hernández, 2009; Verdugo, 2013). La calidad de vida se puede considerar como un constructo social el cual tiene tres dimensiones, la calidad ambiental, el bienestar y la identidad (Hernández, 2009).

"La calidad de vida se debe mirar desde dos puntos: la parte individual (privada) y la parte colectiva (pública); desde lo individual o privado es importante mirar: percepción, sentido de vida, utilidad, valoración, felicidad, satisfacción de necesidades y demás aspectos subjetivos que son difícilmente cuantificables, pero que hacen que una vida tenga calidad con responsabilidad moral" (Cardona y Agudelo, 2005, p. 88).

Otro termino importante a tratar y que va muy relacionado con la calidad de vida es el compromiso que los gobiernos tienen con los ciudadanos, conexo principalmente con las promesas que se hacen de mejorar los servicios públicos de los ciudadanos.

\subsection{Compromiso con el ciudadano}

Es el deber del estado o gobierno el garantizar el bienestar de la sociedad (Ruiz, 2016), es decir, debe atender las diversas necesidades que puedan surgir en la población (Verdugo y Schalock, 2013), por lo cual, constantemente debe asegurarse que todas sus infraestructuras estén en buen estado y que sus servicios sean de la mejor calidad posible (Brewer y Santofimio, 2013), para lo que se cuenta con ciertos estándares internacionales de los requisitos mínimos que deben de tener los servicios ofrecidos por el gobierno, como es el caso de la salud, la distribución de agua, electricidad, drenaje, transporte, entre otros (Aguilar, 2013).

\section{METOdOLOGÍA}

En el presente trabajo se analiza la influencia de la cultura de calidad en la Responsabilidad Social del gobierno municipal de la ciudad de Villaflores, Chiapas (México). Lo que llevó a plantear una pregunta de Investigación de la cual se derivó un objetivo, la cual fue: ¿Cómo influye la Responsabilidad Social Gubernamental en la cultura de calidad del Gobierno municipal de Villaflores, Chiapas?

El objetivo general de este trabajo fue analizar cuál es la Influencia de la Responsabilidad Social Gubernamental en la Cultura de calidad del gobierno de Villaflores, Chiapas (México).

La investigación se guio por una hipótesis generada a partir del objetivo y la revisión de la literatura, de la cual se espera que la actuación responsable del gobierno municipal represente variables de impacto en la percepción del ciudadano hacia la legitimidad, transparencia y calidad de vida, la hipótesis planteada fue la siguiente:

H1: La Responsabilidad Social Gubernamental influye positivamente en la cultura de calidaddel Gobierno Municipal. 
Para la recogida de información se utilizó el cuestionario elaborado y adaptado por Sánchez-Fernández y Ríos-Manríquez (2019) basado en los instrumentos elaborados por Remoaldo et al (2017) y Sánchez-Fernández (2017), del cual se utilizaron las dimensiones de Cultura de Calidad, calidad de vida, Imagen Gubernamental, Creación de empleo y Responsabilidad Social Gubernamental. La operacionalización de las variables consideradas en esta investigación se muestra en la tabla 1.

Tabla 1. Operacionalización de variables.

\begin{tabular}{|c|c|c|c|c|}
\hline Variable & Indicadores & Código & Definición & Referencia teórica \\
\hline \multirow{4}{*}{$\begin{array}{l}\text { Cultura de } \\
\text { calidad }\end{array}$} & $\begin{array}{l}\text { Preocupación } \\
\text { por mejora de } \\
\text { calidad de vida }\end{array}$ & $\mathrm{MCV}$ & $\begin{array}{l}\text { Interés que muestra el gobierno por } \\
\text { mejorar las condiciones en las que } \\
\text { viven los habitantes. }\end{array}$ & $\begin{array}{ll}\text { (Vélez-Romero y } & \text { Cano- } \\
\text { Lara, } & 2016 ; \\
\text { Cardona y Agudelo, 2005; } \\
\text { Hernández, } & 2009 ; \\
\text { Subirats, 1994) } & \end{array}$ \\
\hline & $\begin{array}{l}\text { Creación de } \\
\text { empleo }\end{array}$ & CE & $\begin{array}{l}\text { Es el aumento en el número de } \\
\text { personal ocupado de una población. }\end{array}$ & $\begin{array}{l}\text { (Dolado, García-Serrano, } \\
\text { \& Gómez, 1997). }\end{array}$ \\
\hline & $\begin{array}{l}\text { Formación y } \\
\text { desarrollo } \\
\text { profesional }\end{array}$ & FDP & $\begin{array}{l}\text { Se trata de una fase del desarrollo de } \\
\text { una persona que involucra una } \\
\text { necesidad de superarse asi mismo. }\end{array}$ & $\begin{array}{l}\text { (Ulf-Daniel, 2009;Bowen, } \\
\text { 1996; Daniels, 1992) }\end{array}$ \\
\hline & $\begin{array}{l}\text { lgualdad de } \\
\text { oportunidades }\end{array}$ & 10 & $\begin{array}{l}\text { El Gobierno, tambien debe procurar } \\
\text { que exista igualdad } \\
\text { deoportunidades, tanto en empleo } \\
\text { como en posibilidades de tener una } \\
\text { vida digna. }\end{array}$ & $\begin{array}{l}\text { (Wu, 2015; Ulf-Daniel, } \\
\text { 2009; Levy, 2007) }\end{array}$ \\
\hline \multirow{4}{*}{$\begin{array}{l}\text { Responsabilida } \\
\text { d Social } \\
\text { Gubernamental }\end{array}$} & Transparencia & Trans & $\begin{array}{l}\text { Se conoce como transparencia al } \\
\text { conjunto de disposiciones y medidas } \\
\text { que garantizan la publicidad de la } \\
\text { información relativa de los actos de } \\
\text { las instituciones y el acceso de los } \\
\text { ciudadanos a dicha información. }\end{array}$ & $\begin{array}{l}\text { (Ley de transparencia y } \\
\text { acceso a la información } \\
\text { púbica, 2004; Del hierro, } \\
\text { 2013). }\end{array}$ \\
\hline & $\begin{array}{l}\text { Programas } \\
\text { sociales }\end{array}$ & PS & $\begin{array}{l}\text { Son intervenciones que hacen los } \\
\text { gobiernos para modificar o mejorar } \\
\text { los problemas sociales por los que } \\
\text { está pasando la sociedad. }\end{array}$ & $\begin{array}{l}\text { (Levy, 2007; Cardozo, } \\
\text { 2005) }\end{array}$ \\
\hline & $\begin{array}{l}\text { Limpieza del } \\
\text { municipio }\end{array}$ & LM & $\begin{array}{l}\text { Es la acción que re realiza para quitar } \\
\text { la suciedad y lo perjudicial. }\end{array}$ & $\begin{array}{l}\text { (Vélez-Romero y Cano- } \\
\text { Lara, 2016; Wilson, 2015) }\end{array}$ \\
\hline & $\begin{array}{l}\text { Actuar } \\
\text { conforme a la } \\
\text { ley }\end{array}$ & Ley & $\begin{array}{l}\text { Cumplir con las reglas que se } \\
\text { establecen en un lugar determinado. }\end{array}$ & $\begin{array}{l}\text { (Del Hierro, 2013; } \\
\text { Suchman, 1995; Calduch, } \\
\text { 1991; Meyer y Scott; } \\
\text { 1983) }\end{array}$ \\
\hline \multirow{3}{*}{ Calidad de Vida } & $\begin{array}{l}\text { Servicios } \\
\text { calidad }\end{array}$ & Scal & $\begin{array}{l}\text { Los servicios cumplen los estándares } \\
\text { de calidad internacionales. }\end{array}$ & $\begin{array}{lr}\text { Verdugo, } & \text { 2013; } \\
\text { Hernández, } & \text { 2009; } \\
\text { Rodríguez, 2008; } & \text { Rueda, } \\
\text { 2004) } & \\
\end{array}$ \\
\hline & $\begin{array}{l}\text { Cuidado } \\
\text { ambiental }\end{array}$ & CA & $\begin{array}{l}\text { Las acciones que los gobiernos } \\
\text { toman para proteger los ecosistemas } \\
\text { o recursos naturales. }\end{array}$ & $\begin{array}{lr}\text { Verdugo, } & 2013 ; \\
\text { Hernández, } & 2009 ; \\
\text { Rodríguez, 2008; } & \text { Rueda, } \\
\text { 2004) } & \\
\end{array}$ \\
\hline & $\begin{array}{l}\text { Protección de } \\
\text { derechos }\end{array}$ & PD & $\begin{array}{l}\text { Son las acciones que toman los } \\
\text { gobiernos para proteger y mantener } \\
\text { los derechos humanos de la sociedad } \\
\text { sobre la que gobiernan. }\end{array}$ & $\begin{array}{l}\text { (Council of Europe, 2018; } \\
\text { Bustillos, 2011) }\end{array}$ \\
\hline
\end{tabular}




\begin{tabular}{|c|c|c|c|c|}
\hline \multirow{3}{*}{ Gubernamental } & $\begin{array}{l}\text { Buenos } \\
\text { servicios e } \\
\text { infraestructuras }\end{array}$ & BSEI & $\begin{array}{l}\text { Satisfacción de las necesidades o } \\
\text { expectativas de los ciudadanos. }\end{array}$ & $\begin{array}{lr}\text { (Verdugo, } & 2013 ; \\
\text { Hernández, } & 2009 ; \\
\text { Rodríguez, 2008; } & \text { Rueda, } \\
\text { 2004) } & \end{array}$ \\
\hline & Seguridad & Seg & $\begin{array}{l}\text { Ausencia de peligro en una } \\
\text { población o asentamiento humano. }\end{array}$ & $\begin{array}{l}\text { (Bailey, 2015; Benítez, } \\
\text { 2009; Calleros, 2010) }\end{array}$ \\
\hline & Eficiencia & Efic & $\begin{array}{l}\text { Cumplimiento de los proyectos en el } \\
\text { menor tiempo y con la mejor calidad. }\end{array}$ & $\begin{array}{l}\text { (Gordoa, 2008; Jiménez, } \\
\text { 2004; Mokate, 2001) }\end{array}$ \\
\hline $\begin{array}{l}\text { Compromiso } \\
\text { con el } \\
\text { ciudadano }\end{array}$ & $\begin{array}{l}\text { Cumplimiento } \\
\text { de estándares } \\
\text { de calidad en } \\
\text { sus servicios }\end{array}$ & CEC & $\begin{array}{l}\text { Se cumplen las expectativas en } \\
\text { infraestructura } \\
\text { otorgados. }\end{array}$ & $\begin{array}{lr}\text { (Verdugo, } & \text { 2013; } \\
\text { Hernández, } & \text { 2009; } \\
\text { Rodríguez, 2008; } & \text { Rueda, } \\
\text { 2004) } & \\
\end{array}$ \\
\hline Originario & $\begin{array}{l}\text { Lugar de } \\
\text { nacimiento }\end{array}$ & Or & Tiene su origen en algún lugar. & $\begin{array}{l}\text { (Fernández-Robín } \\
\text { Aqueveque-Torres 2001; } \\
\text { Lamb, Hair, y McDaniel } \\
\text { 2002; Pride y Ferrell, } \\
\text { 1997). }\end{array}$ \\
\hline
\end{tabular}

Fuente: Elaboración propia con base en la literatura y al instrumento elaborado por Sánchez-Fernández y RíosManríquez (2019).

Debido a que los cuestionarios fueron aplicados con una accesibilidad y proximidad limitada se llevó a cabo un muestreo secuencial por cuotas (Otzen y Manterola, 2017).El estudio fue un piloteo de tipo cuantitativo exploratorio correlacional (Véliz, 2011),y de regresión lineal múltiple (Wooldridge, 2010; Gujarati, 2003) en el que participaron 60 personas que habitan en el municipio de Villaflores, Chiapas, segmentados por rangos de edad de acuerdo con el reporte GEM (2015) tal como se muestra en la tabla 2.

Tabla 2. Cuadro Resumen de Metodología seguida.

\begin{tabular}{cc} 
Método de levantamiento & Cara a cara \\
Periodo de levantamiento & Febrero-marzo de 2018. \\
Sujetos de estudio & Personas que vivan en el municipio de Villaflores, Chiapas. \\
Tamaño de muestra (Piloteo) & 60 personas que habitan en la ciudad de Villaflores, Chiapas. \\
Selección de muestra & Sistemático por cuotas. \\
Técnicas de análisis estadístico & Tabla de contingencia, correlacional y regresión lineal \\
Instrumento & Elaborado por Sánchez-Fernández y Ríos-Manríquez (2019). \\
\hline
\end{tabular}

Fuente: Elaboración propia.

Se puede definir la segmentación cómo la división de una muestra en grupos fácilmente identificables y relativamente significativos (Kotler y Armstrong, 2017; Lamb, Hair, y McDaniel, 2002), esta división se fundamenta con las diferencias que existen entre personas tanto individual como grupalmente (Fernández-Robín y Aqueveque-Torres, 2001).

Estas divergencias dan como resultado que la impresión que pueda tener cada sujeto sobre algún tema en específico (Fernández-Robín y Aqueveque-Torres, 2001), varíe dependiendo de alguna de las características sociodemográficas que tengan (Kotler y Armstrong, 2017).

De acuerdo con Pride y Ferrell (1997) si una persona proviene de otro lugar, conoce formas de trabajar y cosas disímiles a las del lugar en el que viva actualmente, lo que puede influir en la percepción sobre que tan bueno o malo es algo que se parctiva normalmente en el lugar (Lamb, et al., 2002). 
De los 60 encuestados el 65\% fueron hombres, 35\% mujeres, el 30\% se encontraban en un rango de edad de 25 a 34 años, y $21.7 \%$ em edades de 18 a 24, mientras que el $53.3 \%$ nació en este municipio y el $46.7 \%$ vienen de otros municipios, el $68 \%$ lleva viviendo en el municipio más de 20 años, y el $31.7 \%$ lleva menos de 20 años habitando en este lugar; el $45 \%$ de los encuestados contaba con licenciatura y $25 \%$ con maestría (ver figura 2 ).

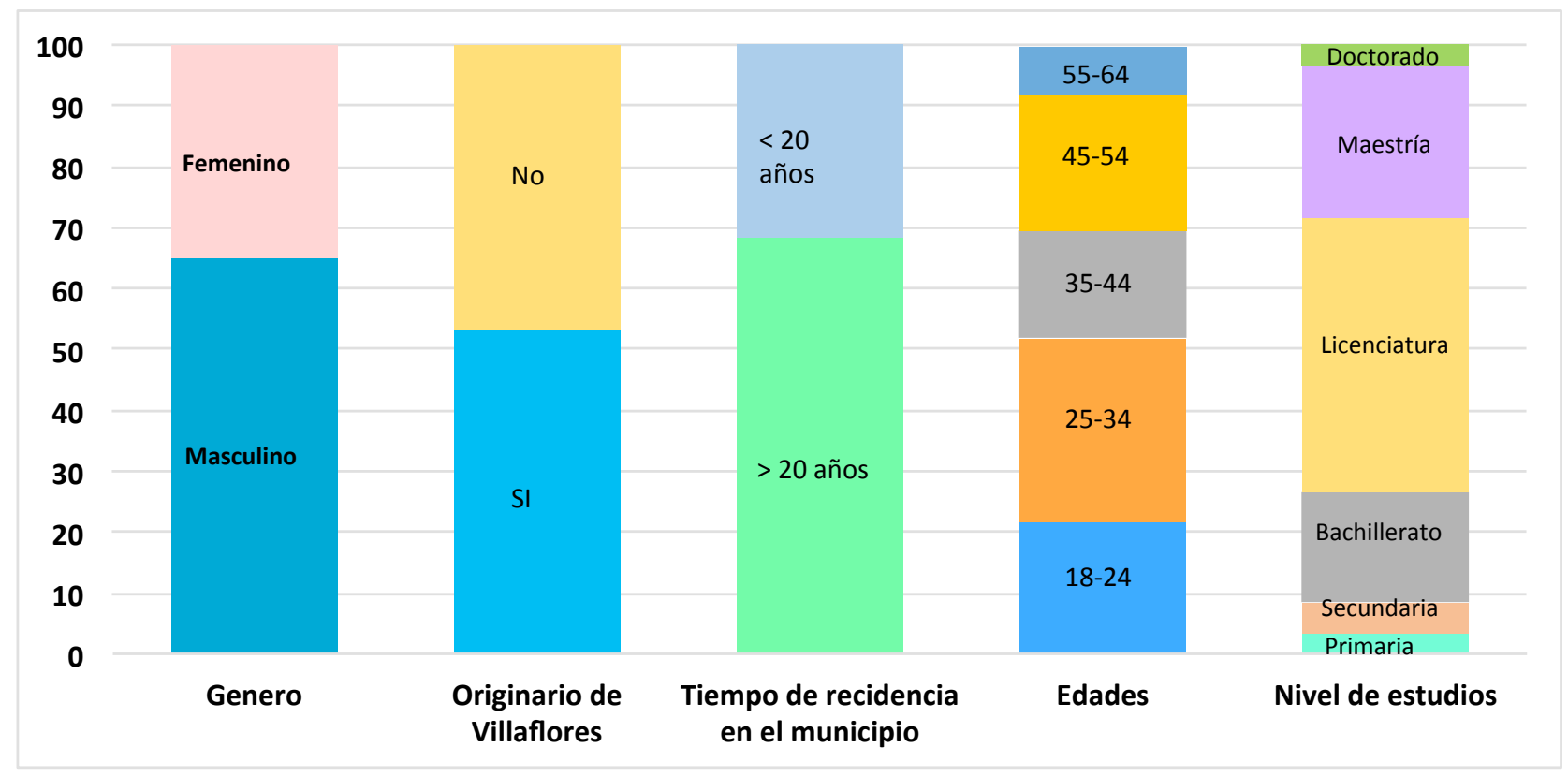

Figura 2. Perfil de los encuestados

Fuente: Elaboración propia con base en los datos obtenidos

De los 60 encuestados el $65 \%$ fueron hombres, 35\% mujeres, el $30 \%$ se encontraban en un rango de edad de 25 a 34 años, y $21.7 \%$ de edades de 18 a 24 y el $22 \%$ en edades de 45 a 54 años, el 18\% de edades de 35 a 44 años y 8\% tenían una edad de entre 55 a 64 años. El 53.3\% nació en este municipio y el $46.7 \%$ vienen de otros municipios, de los encuestados el $68 \%$ lleva viviendo en el municipio más de 20 años, y el 18.3\% lleva menos de 5 años habitando en este lugar. El 45\% de los encuestados contaba con licenciatura, $25 \%$ con maestría, $18.3 \%$ con bachillerato, $5 \%$ con secundaria y $3.3 \%$ contaban con doctorado, teniendo el mismo porcentaje para primaria.

La recolección de datos se llevó a cabo mediante un instrumento con escala Likert (Sáenz y Tamez, 2014), aplicado durante el mes de febrero de 2018. El cuestionario se validó estadísticamente a través del Alfa de Cronbach con un resultado de 0.942 , que confirma la consistencia y confiabilidad del instrumento empleado (Nunnally, 1970; Cohen y Swerdlick, 2001).

Se llevó a cabo un análisis de regresión lineal múltiple a través del método de Mínimos Cuadrados Ordinarios (MCO) dado por Gujarati (2004) y Wooldridge (2010), para el cual se utilizaron las siguientes pruebas: Kolgomorov-Smirnov, Durbin.Watson, Breusch-Pagan para detectar heterocedasticidad y verificar que las variables explicativas del modelo de Cultura de Calidad en el Gobierno son los Mejores Estimadores Lineales Insesgados (MELI), bajo el supuesto de que el modelo es lineal en sus parametros. El modelo planteado para esta investigación es el siguiente:

$$
C d e C_{\imath}=\beta_{0}+\beta_{1} R S G_{i}+\beta_{2} C_{d e} V_{i}+\beta_{3} I G_{i}+\beta_{4} C C_{i}+\varepsilon_{i}
$$


Donde:

$\mathrm{CdeC}_{\mathrm{i}}=$ Cultura de Calidad

$\mathrm{RSG}_{\mathrm{i}}=$ Responsabilidad Social Gubernamental

$\mathrm{CdeV}_{\mathrm{i}}=$ Calidad de Vida

$\mathrm{IG}_{\mathrm{i}}=$ Imagen Gubernamental

$\mathrm{CC}_{\mathrm{i}}=$ Compromiso con el ciudadano

Para el análisis de los datos se utilizó el programa estadístico SPSS v.23 en el análisis de contingencia, el análisis de correlaciones bivariados con el coeficiente de correlación de Pearson ya que se desea evaluar el grado de asociación de las variables de estudio y la regresión lineal, aunque para este último también se utilizó el programa estadístico STATA v.12 (Sáenz y Tamez, 2014).

\section{RESULTADOS Y DISCUSIÓN}

El análisis de los resultados se hizo empleando análisis descriptivo con las medias por dimensión, así como tablas de contingencia, correlaciones de Pearson, y regresión lineal multiple (Sáenz y Tamez, 2014; Wooldridge, 2010;Gujarati, 2003).

\subsection{Descriptivos}

Se encontró que hay una percepción negativa en todas las dimensiones analizadas por parte de los ciudadanos, especialmente en el aspecto del compromiso que tiene el Gobierno municipal con los ciudadanos, lo que está indicando que los ciudadanos no están percibiendo los esfuerzos que el gobierno de Villaflores, Chiapas hace para beneficio de estos (Ver tabla 3).

Tabla 3. Estadísticos Descriptivos

\begin{tabular}{ccc}
\hline Dimensión & Media & Desviación estándar \\
\hline Cultura de calidad & 2.09 & 0.87 \\
Responsabilidad Social Gubernamental & 1.85 & 0.78 \\
Calidad de Vida & 1.94 & 0.79 \\
Imagen Gubernamental & 2.44 & 0.83 \\
con el ciudadano & 1.70 & 0.91 \\
\hline
\end{tabular}

Fuente: Elaboración propia con base en los datos obtenidos.

De igual forma, se tiene una percepción negativa hacia el manejo del gobierno con principios de la cultura de calidad y también hacia que este maneje o implemente la Responsabilidad social.

\subsection{Contingencias}

Como se observa en la tabla 4 , el $\mathbf{2 6 . 7 \%} \%$ de los encuestados nació en el municipio de estudio (Villaflores) y se encuentran totalmente en desacuerdo con que el municipio se maneje bajo los principios de la cultura de calidad, del mismo modo, el 30\% no nació en el municipio y se encuentran totalmente en desacuerdo con que el gobierno se maneje con cultura de calidad. Por 
otra parte, solo el $1.7 \%$ está moderadamente de acuerdo con que el gobierno tenga cultura de calidad.

Tabla 4. Cultura de calidad y origen

\begin{tabular}{cccccc}
\hline $\begin{array}{c}\text { Originario } \\
\text { del } \\
\text { municipio }\end{array}$ & $\begin{array}{c}\text { Totalmente en } \\
\text { desacuerdo }\end{array}$ & $\begin{array}{c}\text { Moderadamente en } \\
\text { desacuerdo }\end{array}$ & Neutral & $\begin{array}{c}\text { Moderadamente } \\
\text { de acuerdo }\end{array}$ & Total \\
\hline Si & $26.7 \%$ & $6.7 \%$ & $20.0 \%$ & $0.0 \%$ & $53.3 \%$ \\
NO & $30.0 \%$ & $6.7 \%$ & $8.3 \%$ & $1.7 \%$ & $46.7 \%$ \\
Total & $56.7 \%$ & $13.3 \%$ & $28.3 \%$ & $1.7 \%$ & 100.0 \\
$\%$
\end{tabular}

Fuente: Elaboración propia con base en los datos obtenidos.

El 56.7\% de los encuestados se encuentran totalmente en desacuerdo con que el municipio esté actuando con Responsabilidad Social, el $28.3 \%$ nació en el municipio y está totalmente en desacuerdo con que el gobierno sea Socialmente Responsable, lo mismo sucede con los no nacidos en el municipio, los cuales representaron el $28.3 \%$ (ver tabla 5), por otra parte, solo el $1.7 \%$ de los encuestados considera que el gobierno sea Socialmente Responsable.

Tabla 5. Responsabilidad Social Gubernamental y origen.

\begin{tabular}{cccccc}
\hline \multirow{2}{*}{$\begin{array}{c}\text { Originario del } \\
\text { municipio }\end{array}$} & \multicolumn{3}{c}{ Responsabilidad Social Gubernamental } & \multirow{2}{*}{ Total } \\
\cline { 2 - 5 } & $\begin{array}{c}\text { Totalmente en } \\
\text { desacuerdo }\end{array}$ & $\begin{array}{c}\text { Moderadamente } \\
\text { en desacuerdo }\end{array}$ & Neutral & $\begin{array}{c}\text { Moderadament } \\
\text { e de acuerdo }\end{array}$ & \\
\hline Si & $28.3 \%$ & $20.0 \%$ & $3.3 \%$ & $1.7 \%$ & $53.3 \%$ \\
NO & $28.3 \%$ & $11.7 \%$ & $6.7 \%$ & $0.0 \%$ & $46.7 \%$ \\
Total & $56.7 \%$ & $31.7 \%$ & $10.0 \%$ & $1.7 \%$ & $100.0 \%$ \\
\hline
\end{tabular}

Fuente: Elaboración propia con base en los datos obtenidos.

El 58.3\% de los entrevistados se encuentra en desacuerdo con tener una buena calidad de vida, por otra parte, el $18.3 \%$ son neutrales o indiferentes en cuanto a si tienen una buena calidad de vida (véase tabla 6).

Tabla 6. La Calidad de Vida y origen

\begin{tabular}{ccccc}
\hline \multirow{2}{*}{$\begin{array}{c}\text { Originario del } \\
\text { municipio }\end{array}$} & $\begin{array}{c}\text { Totalmente en } \\
\text { desacuerdo }\end{array}$ & $\begin{array}{c}\text { Moderadamente } \\
\text { en desacuerdo }\end{array}$ & Neutral & Total \\
\hline Si & $28.3 \%$ & $13.3 \%$ & $11.7 \%$ & $53.3 \%$ \\
NO & $30.0 \%$ & $10.0 \%$ & $6.7 \%$ & $46.7 \%$ \\
Total & $58.3 \%$ & $23.3 \%$ & $18.3 \%$ & $100.0 \%$ \\
\hline
\end{tabular}

Fuente: Elaboración propia con base en los datos obtenidos.

El 30\% de los encuestados se encuentran en desacuerdo con que el gobierno municipal tenga una Imagen Gubernamental Positiva, el $18.3 \%$ de los que si nacieron en Villaflores se encuentra totalmente en desacuerdo con que hay una Imagen positiva del Gobierno del municipio, de igual forma el $11.7 \%$ de los encuestados no nacieron en el municipio y están totalmente en desacuerdo con que el gobierno municipal tenga una buena imagen. No obstante, el $41.7 \%$ están moderadamente en desacuerdo con que el gobierno de Villaflores, Chiapas tenga una imagen positiva (ver tabla 7).

Tabla 7. Imagen Gubernamental y origen 


\begin{tabular}{cccccc}
\hline \multirow{2}{*}{$\begin{array}{c}\text { Originario del } \\
\text { municipio }\end{array}$} & $\begin{array}{c}\text { Totalmente en } \\
\text { desacuerdo }\end{array}$ & $\begin{array}{c}\text { Moderadamente } \\
\text { en desacuerdo }\end{array}$ & Neutral & $\begin{array}{c}\text { Moderadamente } \\
\text { de acuerdo }\end{array}$ & Total \\
\hline Si & $18.3 \%$ & $21.7 \%$ & $11.7 \%$ & $1.7 \%$ & $53.3 \%$ \\
NO & $11.7 \%$ & $20.0 \%$ & $13.3 \%$ & $1.7 \%$ & $46.7 \%$ \\
Total & $30.0 \%$ & $41.7 \%$ & $25.0 \%$ & $3.3 \%$ & $100.0 \%$ \\
\hline
\end{tabular}

Fuente: Elaboración propia con base en los datos obtenidos.

El 56.7\% de los encuestados está completamente en desacuerdo con que el gobierno de Villaflores, Chiapas esté cumpliendo con los compromisos que tienen con ellos, es decir, no creen que el gobierno esté realizando acciones en su beneficio, por otra parte, el $20 \%$ está moderadamente en desacuerdo, mientras que el 3.3\% está moderadamente de acuerdo con que el municipio cumpla con sus compromisos para con los habitantes (ver tabla 8).

Tabla 8. Compromiso con el ciudadano y origen

\begin{tabular}{cccccc}
\hline Originario del & \multicolumn{5}{c}{ Compromiso con el Ciudadano } \\
\cline { 2 - 5 } municipio & $\begin{array}{c}\text { Totalmente en } \\
\text { desacuerdo }\end{array}$ & $\begin{array}{c}\text { Moderadamente } \\
\text { en desacuerdo }\end{array}$ & Neutral & $\begin{array}{c}\text { Moderadamente } \\
\text { de acuerdo }\end{array}$ & Total \\
\hline Si & $28.3 \%$ & $10.0 \%$ & $13.3 \%$ & $1.7 \%$ & $53.3 \%$ \\
NO & $28.3 \%$ & $10.0 \%$ & $6.7 \%$ & $1.7 \%$ & $46.7 \%$ \\
Total & $56.7 \%$ & $20.0 \%$ & $20.0 \%$ & $3.3 \%$ & $100.0 \%$ \\
\hline
\end{tabular}

Fuente: Elaboración propia con base en los datos obtenidos.

En general, en todos los casos, ya sea en términos de la cultura de calidad, la RSG, la imagen gubernamental o el compromiso con el ciudadano, los habitantes sin importar que sean originarios o no del municipio tienen una percepción negativa de lo que su gobierno hace o deja de hacer para beneficio de la sociedad, lo cual está indicando que la situación en el municipio es así y no depende del origen de los encuestados, lo cual coincide con los niveles de significancia encontrados en la Chi-Cuadrado, la cual en todos los casos es superior a 0.05 .

\subsection{Correlaciones}

Analizando si la Responsabilidad Social Gubernamental influye en la Cultura de calidad del gobierno municipal independientemente de si nacieron o no en el municipio se llevó a caboun análisis correlacional de las variables analizadas. Como se observa en la tabla 9, en general todas las variables se relacionan positiva y significativamente con la cultura de calidad; sin embargo, la que mejor se relaciona es la Responsabilidad Social, al tener un índice de correlación de 0.629 y ser significativo a un nivel de 0.01 . Las otras dos variables que mejor se relacionan con la cultura de calidad son la calidad de vida y la Imagen Gubernamental.

Tabla 9. Correlación de Pearson entre cultura de calidad, Responsabilidad Social Gubernamental, Calidad de Vida, Imagen Gubernamental y Compromiso con el ciudadano.

\begin{tabular}{cccccc}
\hline & RSG & CdeV & IG & RC & CdeC \\
\hline Responsabilidad Social Gubernamental (RSG) & 1 & & & & \\
Calidad de Vida (CdeV) & $.601^{* *}$ & 1 & & & \\
Imagen Gubernamental (IG) & $.700^{* *}$ & $.446^{* *}$ & 1 & & \\
Compromiso con el ciudadano (RC) & $.631^{* *}$ & $.401^{* *}$ & $.548^{* *}$ & 1 & \\
Cultura de Calidad (CdeC) & $.629^{* *}$ & $.574^{* *}$ & $.563^{* *}$ & $.436^{* *}$ & 1 \\
\hline
\end{tabular}


* La correlación es significativa en el nivel 0,05 (bilateral).

** La correlación es significativa en el nivel 0,01 (bilateral).

Fuente: Elaboración propia con base en los datos obtenidos.

\subsection{Regresión Lineal}

Se encontró que el modelo tiene un poder explicativo de la varianza de la variable dependiente (Cultura de calidad) del 44.4\% ( $R^{2}$ Ajustada de 0.444 ), quedando el modelo como se muestra en la tabla 9. La prueba $F(12.791)$ con una Prob $>F$ de 0.000 indica que las variables independientes son relevantes para la explicación de la cultura de calidad (ver tabla 10).

Tabla 10.Análisis de regresión múltiple

\begin{tabular}{ll}
\hline \multicolumn{1}{c}{ Variable } & Modelo \\
\hline Responsabilidad Social Gubernamental & $0.32^{*}$ \\
Calidad de vida & $0.326^{* *}$ \\
Imagen Gubernamental & 0.234 \\
Compromiso con el ciudadano & 0.014 \\
Constante & 0.269 \\
Observaciones & 60 \\
$\mathbf{F}$ & $12.791^{* * *}$ \\
Prob>F & 0.000 \\
$\mathbf{R}^{2}$ ajustado & 0.444 \\
Kolmogórov-Smirnov & 0.200 \\
Breuch-Pagan & 0.67 \\
Prob>X & 0.41 \\
Ramsey & 0.89 \\
Prob>F & 0.559 \\
Durbin-Watson & $1.931^{* * *}$ \\
\hline
\end{tabular}

Uno, dos y tres asteriscos indican niveles de significancia del 10\%, 5\% y $1 \%$ respectivamente. Fuente: Elaboración propia con base en resultados de SPSS.

Se aplico la prueba de Kolgomorov-Smirnov para saber si los datos se comportaban de manera normal, aplicando la HO: Los datos no difieren de una distribución normal. Con la corrección de Lilliefors se obtuvo una significancia asintótica de 0.2 por lo que se conserva la HO y por lo tanto los datos se comportan conforme a una distribución normal. De igual forma se hizo la prueba de Durbin-Watson para saber si había correlación entre las variables, para esta prueba se obtuvo un valor de 1.931, por lo que podemos decir que no hay evidencia estadística suficiente de que los términos de error estén auto correlacionados positivamente.Se aplicó la prueba de Ramsey para asegurar que no hubiese ninguna variable excluida en el modelo, con una Prob>F de 0.559 se puede corroborar que no hay ninguna variable excluida (ver tabla 10).

Con la finalidad de verificar que en el modelo no se tengan problemas de multicolinealidad entre las variables independientes se aplicaron las pruebas de Factor de Inflación de Varianzas (FIV) y la prueba de tolerancia (TOL), los resultados de dichas pruebas se muestran en la tabla 11, en la cual se puede observar que todas las variable explicativas presentan un FIV menor a 10 y una TOL mayor a 0.1 lo que nos indica que no hay problemas de multicolinealidad.

Tabla 11. Indicadores de multicolinealidad del modelo de cultura de calidad en el gobierno.

\begin{tabular}{ccc}
\hline \multirow{2}{*}{ Variable } & \multicolumn{2}{c}{ Estadísticos de Colinealidad } \\
\cline { 2 - 3 } & Tolerancia & FIV \\
\hline
\end{tabular}




\begin{tabular}{ccc}
\hline Responsabilidad Social Gubernamental & .352 & 2.845 \\
Calidad de vida & .637 & 1.570 \\
Imagen Gubernamental & .491 & 2.037 \\
Compromiso con el ciudadano & .579 & 1.726 \\
\hline
\end{tabular}

Fuente: Elaboración propia con base en los datos obtenidos.

De igual forma se verificó si había homocedasticidad, utilizando la prueba de BreuschPagan, con la finalidad de verificar que las variables predictoras son los mejores estimadores lineales, tal como se observa en la tabla 9, no existe homocedasticidad en el modelo.

Finalmente, el modelo econométrico determinado en este trabajo de la Cultura de Calidad en el gobierno municipal queda de la siguiente forma:

$$
C_{d e C_{\imath}}=0.269+320 R S G_{i}+0.326 C_{d e V_{i}}+0.234 I G_{i}+0.014 C C_{i}
$$

Debido a que el modelo pasó satisfactoriamente todas las pruebas se puede decir que hay evidencia estadística suficiente para aceptar este modelo explicativo de Cultura de Calidad en el gobierno municipal.

\section{CONCLUSIONES}

El que las personas tengan acceso a los servicios públicos más básicos; como un buen servicio de transporte público, agua potable, drenaje, el que vivan en un lugar seguro y limpio, que tengan una vida profesional satisfactoria(búsqueda de empleo, balance vida-trabajo, trabajos bien remunerados, etc.); a que el gobierno se preocupe por las cuestiones ambientales que le atañen, como el cuidar los niveles de contaminación; el que la sociedad reconozca los esfuerzos del gobierno y que este actúe de acuerdo a la ley; que los municipios divulguen la información sobre lo que se hace con los recursos y que haga uso adecuado de estos, conforman la cultura de calidad en el gobierno y la Responsabilidad Social Gubernamental, a los cual se les ha reconocido como factores importantes del actuar de los gobiernos, ya que los factores mencionados ayudan a mejorar la imagen del gobierno, a generar confianza y a que un gobierno sea legítimo.

El objetivo de esta investigación que fue analizar cuál es la Influencia de la Responsabilidad Social Gubernamental en la cultura de calidad el gobierno de Villaflores, Chiapas. Se logró y se acepta la hipótesis de trabajo H1 "la Responsabilidad Social Gubernamental influye positivamente en la cultura de calidad del Gobierno Municipal”. Ya que la variable RSG analizada en la regresión lineal tiene una influencia positiva con la cultura de calidad.

En el caso de calidad de vida, se observó la tendencia de ir de moderadamente en desacuerdo a neutral; en términos generales el $58 \%$ de los encuestados está completamente en desacuerdo a tener una buena calidad de vida, esto coincide con los resultados del INEGI (2018), en los indicadores de bienestar y calidad de vida, en los que, para el estado de Chiapas el acceso a servicios de salud lo ubica en el lugar 19/32 y entre los últimos tres lugares en viviendas que cuentan con servicios públicos a nivel nacional. De igual forma se encontró que los encuestados tienen una percepción negativa sobre que el Gobierno sea Socialmente Responsable y que esté procurando cumplir los compromisos que tiene con los ciudadanos, lo cual, tal como lo señalan López-Pinto (2001)y Abreu (1998), si estos factores son descuidados, la imagen que tendran los gobernantes entre los ciudadanos será negativa. 
Se encontró que el gobierno del estado de Chiapas implementó diferentes programas para mejorar la situación de las empresas y el emprendedurismo en el estado, así como un programa de mejora a la vivienda implementado en el 2018(Secretaría de Economía y del Trabajo Estado de Chiapas, 2018);sin embargo, en el Portal de Transparencia del estado de Chiapas (2018) no se encuentra disponible informaciónsobre el recurso utilizado, el impacto de los programas, etc. Esto es un foco de alerta para el gobierno, ya que si los ciudadanos no tienen acceso a esta información se puede afectar la imagen gubernamental que manejan, comparando esto con los resultados obtenidos, se observa que los ciudadanos de Villaflores no perciben una mejora en su calidad de vida, lo que está implicando que probablemente estos programas estatales no les estén llegando o no tienen la amplitud necesaria para llegar a más personas.

En cuanto a las correlaciones, se destaca que todas las variables tienen una relación positiva y significativa con la cultura de calidad, sin embargo, la variable que mejor se correlaciona es la Responsabilidad Social Gubernamental, mientras que, la que tiene un índice de correlación más bajo es la de Compromiso con el Ciudadano, esto puede ser debido a que, si bien la mayoría de respuestas indican estar en desacuerdo, cerca del $20 \%$ de las respuestas fueron neutrales, esto pudo ser causado debido a la amplitud del tema, el cual tiene menos indicadores en comparación con la Cultura de Calidad, la Responsabilidad Social Gubernamental o la Calidad de Vida, las cuales debido a la amplitud de sus temas contenían un mayor numero de indicadores.

La aportación principal de esta investigación fue la propuesta del modelo generado a través del análisis econométrico que relaciona a la cultura de calidad en el gobierno con la Responsabilidad Social Gubernamental. El modelo presentado destaca que las variables presentadas son relevantes para explicar a la cultura de calidad en el gobierno, aunado a que no se presentaron problemas de multicolinealidad ni heterocedasticidad.

La limitante de este estudio es que se trató de una prueba piloto, por lo que hace falta ampliar la investigación, sugiriendo ampliar la muestra y posiblemente el área que abarca el estudio, así como profundizar más en las pruebas estadísticas para corroborar lo que ha resultado de esta investigación. De igual forma se propone seguir realizando este tipo de estudios en Villaflores, Chiapas, principalmente debido a la falta de información respecto a estos temas en dicho municipio.

La corrupción es un tema importante por profundizar, al menos en el estado de Chiapas, debido a que la percepción de la transparencia por parte de los ciudadanos puede ser afectada por temas de corrupción y esto a su vez generar desconfianza ciudadana hacia los gobiernos.

\section{BIBLIOGRAFÍA}

Abreu, I. (1998). Líderes e imagen pública en Venezuela: elementos para el estudio del liderazgo. Caracas, Venezuela: Fondo Editorial de Humanidades y Educación.

Aceves, F. d. (2002). Los medios y la construcción de la imagen pública: las elecciones presidenciales de 1994 en México. Cd. México, México: Universidad de Guadalajara. 
Aguilar, A. (2013). Panorama de la Responsabilidad Social en México. México: ResponSable.

Anzola M, O. I. (2005). Caracterizacion de la cultura corporativa. Sotavento MBA, 30-44. Recuperado el 19 de Marzo de 2018, de https://dialnet.unirioja.es/descarga/articulo/5137598.pdf

Bailey, J. (2015). Crimen e impunidad, las trampas de la seguridad en México. Cd. México: DEBATE.

Baldi, G., \& García, E. (2005). Calidad de vida y medio ambiente. La psicología ambiental. Universidades, 9-16.

Barney, J. (1986). Organizational culture: can it be a source of sustained competitive advantage? Academy Of Management Review, 656-665.

Benítez, R. (2009). La Iniciativa Mérida: nuevo paradigma en la relación de seguridad México-Estados Unidos-Centroamérica. Revista Mexicana de Política Exterior, 215-242.

Bowen, P. W. (1996). The need for quality cultures. Training for Quality , 14-18.

Brewer, A.-R., \& Santofimio, J. O. (2013). Control de Convencionalidad y Responsabilidad Del Estado. Bogotá, Colombia: Universidad del Externado de Colombia.

Bustillos, J. (2011). Derechos humanos y protección constitucional. Breve estudio sobre el matrimonio entre personas del mismo sexo en México y en perspectiva comparada. Boletín mexicano de derecho comparado, 1017-1045.

Cajiga C., J. F. (1 de Mayo de 2018). CEMEFI. Obtenido de El concepto de Responsabilidad Social Empresarial: https://www.cemefi.org/esr/images/stories/pdf/esr/concepto_esr.pdf

Calduch, R. (1991). Relaciones Internacionales. Madrid: Edit. Ediciones Ciencias Sociales.

Calleros, J. C. (2010). El vínculo entre seguridad nacional y migración en México. Revista mexicana de política exterior, 9-43.

Cancino del Castillo, C., \& Morales Parragué , M. (2008). Responsabilidad Social Empresarial. Universidad de Chile, 1-58.

Cardona A., D., \& Agudelo G., H. B. (2005). Construcción cultural del concepto calidad de vida. Revista Facultad Nacional de Salud Pública, 70-90.

Cardozo, M. I. (2005). Neoliberalismo y eficiencia de los programas sociales en México. Política y cultura, 169-186.

CENTRARSE. (15 de Abril de 2018). CENTRARSE en Guatemala. Obtenido de ¿Qué es RSE?: http://centrarse.org/?page id=296

Chiapas Gobierno del Estado. (9 de febrero de 2019). Gobierno del Estado de Chiapas. Obtenido de Conoce Chiapas: http://www.chiapas.gob.mx/ubicacion/

Cohen, R. \&. (2001). Pruebas y evaluaciones psicológicas. Introducción a las pruebas y a la medición. México: McGraw Hill. 
Council of Europe. (25 de octubre de 2018). Protección legal de los derechos humanos. Obtenido de COMPASS: Manual de Educación en los Derechos Humanos con Jovenes: https://www.coe.int/es/web/compass/legal-protection-of-human-rights

Daniels, S. (1992). The total quality culture. Work Study, 13-15. doi:10.1108/EUM0000000002675

del Hierro, J. L. (2013). Legitimidad y transparencia. Eunomía. Revista en Cultura de la Legalidad , 179186.

Diez, E. (2007). La cultura y la Responsabilidad Social: Binomio Estratégico en las organizaciones. Visión Gerencial, 231-244.

Dolado, J., García-Serrano, C., \& Gómez, R. (1997). Creación y Destrucción de Empleo: Una Panorámica con nuevos resultados para España. Papeles de Economía Española, 2-29.

Emond, B., \& Taylor, J. Z. (2018). The importance of measuring food safety and quality culture: results from a global training survey. Worldwide Hospitality and Tourism Themes, 369-375. doi:https://doi.org/10.1108/WHATT-02-2018-0012

Fernández-Robín, C., \& Aqueveque-Torres, C. (2001). Segmentación de mercados: buscando la correlación entre variables sicológicas y demográficas. Revista Colombiana de Marketing, 1-14.

García-Álvarez, C. M. (2006). Una aproximación al concepto de cultura organizacional. Universitas Psychologica, 163-174.

GEM. (2015). GEM Mexico 2015-National Report. Monterrey: GEM. Recuperado el Abril de 2018, de https://www.gemconsortium.org/report/49639

Geoff, B. (1997). Leadership and the development of quality culture in schools. International Journal of Educational Management, 52-64.

Gillies, A. C. (2015). Tools to support the development of a quality culture in a learning organisation. The TQM Journal, 471 - 482. doi:http://dx.doi.org/10.1108/TQM-03-2015-0039

González-Ortiz, J. H., Escalera-Chávez, M. E., \& Pérez-Veyna, Ó. (2010). Construcción del cuestionario para determinar el nivel de Cultura de Calidad en MiPymes. Investigación y Ciencia, 39-48.

Gordoa, V. (1999). El Poder de la Imagen Pública. Cd. México: Editores Asociados Mexicanos.

Gordoa, V. (2008). La Imagen Vendedora. Cd. México: Grijalbo.

Gujarati, D. N. (2003). Econometría. Cd. México, México: McGraw-Hill Interamericana.

Hairuddin, M., \& Mohammed, B. M. (2012). Investigation of Malaysian higher education quality culture and workforce performance. Quality Assurance in Education, 289-309. doi:https://doi.org/10.1108/09684881211240330

Hernández Aja, A. (2009). Calidad de vida y medio ambiente urbano. Indicadores locales de sostenibilidad y calidad de vida urbana. Revista invi , 79-11. 
Hernández Juncol, V., Quintana Tápanes, L., Mederos Torres, R., Guedes Díaz, R., García Gutiérrez, B. N., \& Cabrera Rosell, P. (2015). Influencia de la cultura organizacional en la calidad del servicio en el Hospital "Mario Muñoz Monroy". Trabajos Originales, 1-8. Recuperado el 6 de marzo de 2018, de http://scielo.sld.cu/pdf/mil/v37n3/mil03308.pdf

INEGI. (7 de Diciembre de 2016). INEGI. Obtenido de Estadisticas a propósito del día internacional contra la corrupción http://www.inegi.org.mx/saladeprensa/aproposito/2016/corrupcion2016_0.pdf

INEGI. (16 de Mayo de 2018). PIB y Cuentas Nacionales . Obtenido de PIB - Entidad Federativa, anual: www.inegi.org.mx/est/contenidos/proyectos/cn/pibe/

INEGI. (16 de Mayo de 2018). Sistemas de indicadores. Obtenido de Indicadores de Bienestar por entidad federativa: http://www.beta.inegi.org.mx/app/bienestar/\#grafica

ISO. (11 de Junio de 2010). ISO26000. Recuperado el 4 de febrero de 2018, de ISO26000:2010: https://www.iso.org/obp/ui\#iso:std:iso:26000:ed-1:v1:es

Jiménez, R. E. (2004). Indicadores de calidad y eficiencia de los servicios hospitalarios. Una mirada actual. Revista Cubana de Salud Pública. Obtenido de http://scielo.sld.cu/scielo.php?pid=S086434662004000100004\&script=sci_arttext\&tlng=pt

Kotler, P., \& Armstrong, G. (2017). Fundamentos de marketing. Ciudad de México, México: Pearson Educación de México.

Lamb, C., Hair, J., \& McDaniel, C. (2002). Marketing (6ta ed.). Ciudad de México, México: International Thomson Editores.

Levy, S. (2007). ¿PUEDEN LOS PROGRAMAS SOCIALES DISMINUIR LA PRODUCTIVIDAD Y EL CRECIMIENTO ECONÓMICO?: Una hipótesis para México . El Trimestre Económico, 491-540 .

Ley general de Transparencia y Acceso a la Información Pública. (2004). Ley de Transparencia y Acceso a la Información Pública. Ciudad de México: Diario oficial la gaceta.

Lo, T. Y. (2002). Quality culture: a product of motivation within organization. Managerial Auditing Jour, 272-276.

Lomnitz-Adler, C. (2000). Vicios públicos, virtudes privadas: la corrupción en México. Ciudad de México: Porrua.

López-Pinto, B. (2001). La esencia del Marketing. Cataluña, España: EDICIONS UPC.

Maquiavelo, N. (1999). El principe. Florencia: Espasa*Calpe.

Meyer, J., \& Scott, W. R. (1983). Centralization and the legitimacy problems of local government. Organizational Environments : Ritual and Rationality, 199-215.

Mokate, K. M. (2001). Eficacia, eficiencia, equidad y sostenibilidad: ¿Qué queremos decir? Washinton, EUA: BANCO INTERAMERICANO DE DESARROLLO. Serie de documentos de trabajo I-24. 
Moneva, J. M. (2005). Información sobre responsabilidad social corporativa: situación y tendencias. REVISTA ASTURIANA DE ECONOMÍA, 43-69.

Morris, S. D. (1992). Corrupción y política en el México contemporáneo. Ciudad de México: Siglo Veintiuno editores.

Navarro-Galera, A., Ruiz-Lozano, M., de los Ríos-Berjillos, A., \& Tirado-Valencia, P. (2014). La responsabilidad social corporativa en los gobiernos locales: el caso de Reino Unido e Irlanda. Revista Innovar journal, 89-105.

Nunnally, J. (1970). Introduction to psychological measurement. Japon: McGraw Hill.

OMS. (1997). MEASURING QUALITY OF LIFE. OMS, 1-13.

Otzen, T., \& Manterola, C. (2017). Técnicas de Muestreo sobre una Población a Estudio. International Journal Morphol, 227-232.

Portal de Transparencia del Estado de Chiapas. (2018). Portal de Transparencia. Obtenido de Información Pública

Obligatoria:

http://transparencia.chiapas.gob.mx/PortalGral/PortalConsultaFracciones

Pride, W., \& Ferrell, O. (1997). Marketing: conceptos y estrategias (9na ed.).Bogotá, Colombia: McGraw-Hill Interamericana.

Remoaldo, P., Freitas, I., Matos, O., Lopes, H., Silva, S., Sánchez Fernández, M., Cadima Ribeiro, J., \& Ribeiro, V. (2017). The Planning of Tourism on Rural Areas: The Stakeholders' Perceptions of the Boticas Municipality (Northeastern Portugal), European Countryside, 9(3), 504-525. doi: https://doi.org/10.1515/euco-2017-0030

Rivero, P. (2006). La Responsabilidad Social Corporativa en la PYMES. Lan Harremanak, 77-91.

Rodríguez, A. d. (2008). Responsabilidad social empresarial, calidad de vida y trabajo social. Trabajo Social, 0123-4986.

Rueda, S. (2004). Habitabilidad y calidad de vida. Cuadernos de Investigación urbanística, 29-34.

Ruiz, W. (2016). Responsabilidad del estado y sus regímenes. Bogotá, Colombia: Ecoe Ediciones.

Ruiz-Rico, C. (2014). RESPONSABILIDAD SOCIAL PÚBLICA Y CALIDAD DEMOCRÁTICA. Derecho y Cambio Social, 1-17.

Sáenz, K., \& Tamez, G. (2014). Métodos y técnicas cualitativas y cuantitativas aplicables a la investigación en ciencias sociales. Ciudad de México: Editorial Tirant Humanidades.

Sánchez-Fernández, M.D. (2017). Instrumento de medición para el turismo basado en la inclusión, la sostenibilidad, el género y la naturaleza. In Martínez-Fernández, Juanatey-Boga, Viñán-Merecí, Ludeña-Reyes, Membiela-Pollán (Eds) Serie de Documento de Trabajo de Turismo Comunitario. Zona fronteriza Ecuador - Perú. Pp.1-27. 
Sánchez-Fernández, M.D. \& Ríos-Manríquez, M. (2019). Gestión de la Responsabilidad Social en el Gobierno Municipal en el área de turismo. España: RENUTEG \& Universidade da Coruña \& Universidad de Guanajuato.

Subirats, J. (1994). ANALISIS DE POLITICAS PUBLICAS Y EFICACIA DE LA ADMINISTRACION. Madrid, España: MAP.

Suchman, M. (1995). Managing legitimacy: strategic and institutional approaches. Academy of Management Review, 571-610.

71. Ulf-Daniel, E. (2009). Understanding quality culture. Quality Assurance in Education, 343-363. doi:https://doi.org/10.1108/09684880910992322

Vélez-Romero, X. A., \& Cano-Lara, E. D. (2016). Los diferentes tipos de responsabilidad social y sus implicaciones éticas. Revista Científica Dominio de las Ciencias, 117-126.

Véliz Capuñay, C. (2011). Estadistica para administracion y negocios. Naucalpan de Juarez, México.: Pearson.

Verdugo, M. Á., \& Schalock, R. L. (2013). Discapacidad e inclusió. Manual para la docencia .Salamanca: Amarú.

Wilson , F. (2015). The Quality Maturity Model: your roadmap to a culture of quality . Library Management, 258-267.

Wooldridge, J. M. (2010). Introducción a la Econometría un enfoque moderno. México: CENGAGE Learning.

$\mathrm{Wu}, \mathrm{S}$. J. (2015). The impact of quality culture on quality management practices and performance in Chinese manufacturing firms. International Journal of Quality \& Reliability Management, 799-814. doi:https://doi.org/10.1108/IJQRM-12-2013-0199 